\title{
The Scattering and Bound States of the Schrödinger Particle in Generalized Asymmetric Manning-Rosen Type Potential
}

\author{
Ahmet Taş, Soner Alpdoğan, and Ali Havare \\ Department of Physics, Mersin University, 33343 Mersin, Turkey \\ Correspondence should be addressed to Ahmet Taş; aahmet.tas@gmail.com \\ Received 2 July 2014; Accepted 21 August 2014; Published 7 September 2014 \\ Academic Editor: Shi-Hai Dong
}

Copyright (c) 2014 Ahmet Taş et al. This is an open access article distributed under the Creative Commons Attribution License, which permits unrestricted use, distribution, and reproduction in any medium, provided the original work is properly cited. The publication of this article was funded by SCOAP $^{3}$.

\begin{abstract}
We solve exactly one-dimensional Schrödinger equation for the generalized asymmetric Manning-Rosen (GAMAR) type potential containing the different types of physical potential that have many application fields in the nonrelativistic quantum mechanics and obtain the solutions in terms of the Gauss hypergeometric functions. Then we determine the solutions for scattering and bound states. By using these states we calculate the reflection and transmission coefficients for scattering states and achieve a correlation that gives the energy eigenvalues for the bound states. In addition to these, we show how the transmission and reflection coefficients depend on the parameters which describe shape of the GAMAR type potential and compare our results with the results obtained in earlier studies.
\end{abstract}

\section{Introduction}

One of the most important issues discussed in physics is to understand the structures of nucleus, atoms, molecules, and the material objects. Therefore it is important to create models, which contain the potential concept, describing the interactions between the two objects. Some of the potential models have been identified in order to describe the interactions in the nuclei and nuclei-particle and structures of the diatomic and polyatomic molecules. Some of these potentials are called as follows: the Kratzer, Morse, Eckart, RosenMorse, Manning-Rosen, Pöschl-Teller, Hulthen, WoodsSaxon, Scarf, Schiöberg, Deng-Fan, and Cusp potentials [113]. The other important and current topic is to obtain the exact solutions, which describe the scattering and bound states, of the Schrödinger equation that is known as the fundamental equation of nonrelativistic quantum mechanics in the existence of an external potential [14-40]. In order to get complete information about a quantum mechanical system under consideration, one needs to study the scattering and bound states. In [17], the authors have presented the exact solutions which describe $s$-wave scattering states for the Schrödinger equation with the Manning-Rosen potential via the standard method. Ikhdair and Sever have suggested a new approximated scheme to centrifugal term to achieve the $l \neq 0$ solutions of the Schrödinger equation for the Manning-Rosen potential by using the Nikiforov-Uvarov method and they have obtained the corresponding normalized wave functions in terms of the Jacobi polynomial. They have also calculated the bound state energies of various states for $\mathrm{HCl}, \mathrm{LiH}$, and CO diatomic molecules [26]. In the presence of the Hulthen potential, approximate analytical solutions of the radial Schrödinger equation with $l \neq 0$ have been presented by using the Exact Quantization Rule in [27]. Arda et al. have solved the one-dimensional Schrödinger equation for the asymmetric Hulthen potential [28]. In this study they have obtained the scattering and bound states solutions in terms of the hypergeometric functions. In [30], the writers have acquired the energy eigenvalues of the bound states and the corresponding eigenfunctions of the generalized WoodsSaxon potential. Arda et al. have achieved the scattering solutions of the one-dimensional Schrödinger equation with the position-dependent mass in the existence of the WoodsSaxon potential [32]. For the modified Pöschl-Teller potential, the approximation solutions of the Schrödinger equation in one dimension have been obtained with an approximation of the centrifugal term by Agboola [33]. In this work the author has obtained some expectation values using the 


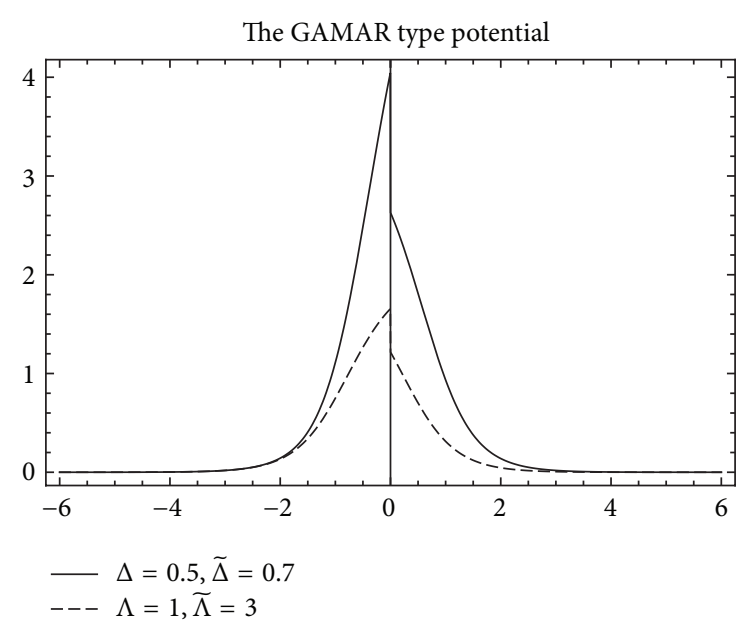

(a)

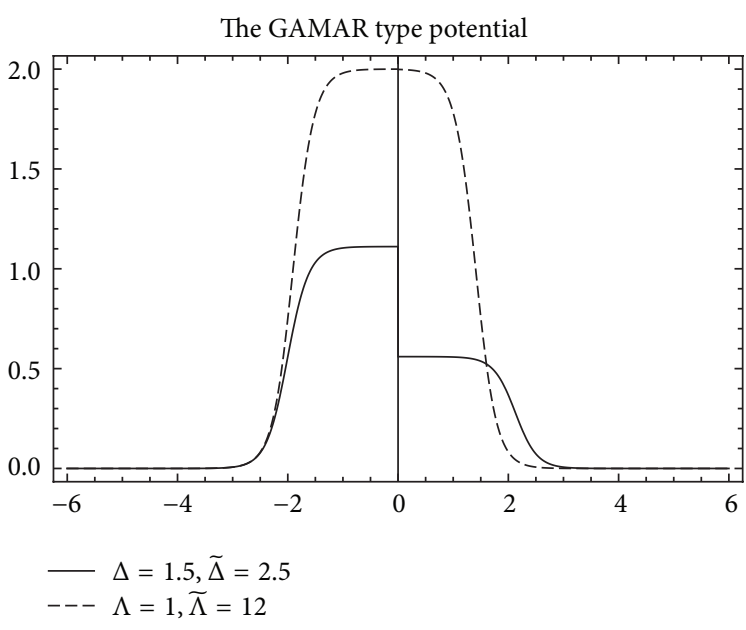

(b)

Figure 1: The shapes of the GAMAR type potential for different values of the potential parameters. (a) shows $\alpha=\beta=2, x_{0}=\tilde{x}_{0}=1$, and $A=B=C=D=1$ (lower values of the $\alpha x_{0}, \beta \widetilde{x}_{0}$ ). (b) shows $\alpha=\beta=5, x_{0}=\widetilde{x}_{0}=2$, and $A=B=C=D=1$ (large values of the $\alpha x_{0}, \beta \tilde{x}_{0}$ ).

Hellmann-Feynman method. Qiang et al. have carried out the approximately scattering states solutions of the $l$-wave Schrödinger equation for the second Pöschl-Teller-like potential by taking a new approximation scheme to the centrifugal term [34]. Tezcan and Sever have obtained the exact solution of the Schrödinger equation for the Rosen-Morse and Scarf potentials with position-dependent mass by using the general point of the canonical transformation [36]. Analytical solutions of the Schrödinger equation with the Makarow and ring-shaped Hartmann potentials for any $n$ and $l$ (states) quantum numbers have been presented by using the asymptotic iteration method in [40].

The Manning-Rosen potential was first proposed to define the vibrational behavior of diatomic molecules by Manning and Rosen in 1933 [5]. Afterwards, it has been used to describe the interactions between two atoms in a diatomic molecule and also it is very reasonable in describing such interactions close to the surface [41-43]. Some of the potentials can be generalized to describe the interactions consisting of more than one process. Therefore, in our study, we have defined the generalized asymmetric Manning-Rosen (GAMAR) type potential which is the similar type of the Manning-Rosen potential, in the following form [44]:

$$
\begin{aligned}
V(x)= & \theta(-x)\left[\frac{A e^{2 \alpha\left(x+x_{0}\right)}}{\left(\Lambda+\Delta e^{\alpha\left(x+x_{0}\right)}\right)^{2}}+\frac{B e^{\alpha\left(x+x_{0}\right)}}{\Lambda+\Delta e^{\alpha\left(x+x_{0}\right)}}\right] \\
& +\theta(x)\left[\frac{C e^{-2 \beta\left(x-\tilde{x}_{0}\right)}}{\left(\widetilde{\Lambda}+\widetilde{\Delta} e^{-\beta\left(x-\tilde{x}_{0}\right)}\right)^{2}}+\frac{D e^{-\beta\left(x-\tilde{x}_{0}\right)}}{\widetilde{\Lambda}+\widetilde{\Delta} e^{-\beta\left(x-\tilde{x}_{0}\right)}}\right],
\end{aligned}
$$

where $\theta(x)$ is the Heaviside step function. All of the parameters in the potential are real. The shape of the GAMAR type potential varies according to the values of the parameters. If
$A, B, C$, and $D$ are positive, it becomes a potential barrier. When $A, B, C$, and $D$ are negative values, it takes into a potential well. The GAMAR type potential form is displayed in Figure 1. In special cases, it reduces to potentials such as the Manning-Rosen, generalized Wood-Saxon (GAWS) [45], Woods-Saxon, asymmetric Hulthen (ASH) [46], Hulthen, asymmetric Cusp (ASC) [47], and Cusp, potentials that have many applications in the relativistic and nonrelativistic quantum mechanics. The special cases of the GAMAR type potential are displayed in Table 1.

The content of this study is arranged as follows: in Section 2, the Schrödinger equation with the GAMAR type potential barrier is solved, the solutions are written in terms of the hypergeometric functions, and the asymptotic behaviors of the solutions are obtained. In the same section, by using the continuity conditions of the wave function and its derivative, the transmission and reflection coefficients are calculated. In Section 3, we get a condition that gives the energy eigenvalues for a Schrödinger particle in the GAMAR type potential well. Finally, we discuss the results in Section 4.

\section{Scattering States and Finding Coefficients of Reflection and Transmission}

The stationary Schrödinger equation in one dimension for a particle with mass $m$ and energy $E$ moving in a external potential is written as the following form (in natural units $\hbar=1)$ :

$$
\left\{\frac{d^{2}}{d x^{2}}+2 m[E-V(x)]\right\} \psi(x)=0 .
$$

To determine scattering states occurring as a result of interaction particles with the GAMAR type barrier potential, 
TABLE 1: The special potentials which are derived from the GAMAR type potential.

\begin{tabular}{|c|c|c|}
\hline The potentials & Varying parameters & The shape of the potential \\
\hline The Manning-Rosen & $\begin{array}{l}A=B=0,0<x<\infty \\
\Lambda=\widetilde{\Lambda}=1, x_{0}=\widetilde{x}_{0}=0 \\
\Delta \rightarrow-1, \widetilde{\Delta} \rightarrow-1, \alpha=\beta\end{array}$ & $V(x)=\frac{C}{\left(e^{\beta x}-1\right)^{2}}-\frac{D}{e^{\beta x}-1}$ \\
\hline The GAWS & $A=C=0, B=D=V_{0}$ & $V(x)=V_{0}\left[\frac{\theta(-x)}{\Delta+\Lambda e^{-\alpha\left(x+x_{0}\right)}}-\frac{\theta(x)}{\widetilde{\Delta}+\widetilde{\Lambda} e^{\beta\left(x-\tilde{x}_{0}\right)}}\right.$ \\
\hline The Woods-Saxon & $\begin{array}{l}A=C=0, B=D=V_{0} \\
\Delta=\widetilde{\Delta}=1, \Lambda=\widetilde{\Lambda}=1 \\
\alpha=\beta, x_{0}=\tilde{x}_{0}\end{array}$ & $V(x)=V_{0}\left[\frac{\theta(-x)}{1+e^{-\alpha\left(x+x_{0}\right)}}-\frac{\theta(x)}{1+e^{\alpha\left(x-x_{0}\right)}}\right]$ \\
\hline The ASH & $\begin{array}{l}A=C=0, B=D=V_{0} \\
\Delta \rightarrow-\Delta, \Lambda=\widetilde{\Lambda}=1 \\
\widetilde{\Delta} \rightarrow-\widetilde{\Delta}, x_{0}=\widetilde{x}_{0}=0\end{array}$ & $V(x)=V_{0}\left[\frac{\theta(-x)}{e^{-\alpha x}-\Delta}-\frac{\theta(x)}{e^{\beta x}-\widetilde{\Delta}}\right]$ \\
\hline The Hulthen & $\begin{array}{l}A=C=0, B=D=V_{0}, \alpha=\beta \\
\Delta \rightarrow-\Delta, \Lambda=\widetilde{\Lambda}=1 \\
\widetilde{\Delta} \rightarrow-\widetilde{\Delta}, x_{0}=\widetilde{x}_{0}=0\end{array}$ & $V(x)=V_{0}\left[\frac{\theta(-x)}{e^{-\alpha x}-\Delta}-\frac{\theta(x)}{e^{\alpha x}-\widetilde{\Delta}}\right]$ \\
\hline The ASC & $\begin{array}{l}A=C=0, B=D=V_{0} \\
\Delta \rightarrow 0, \widetilde{\Delta} \rightarrow 0 \\
x_{0}=\tilde{x}_{0}=0, \Lambda=\widetilde{\Lambda}=1\end{array}$ & $V(x)=V_{0}\left[\frac{\theta(-x)}{e^{-\alpha x}}-\frac{\theta(x)}{e^{\beta x}}\right]$ \\
\hline The Cusp & $\begin{array}{l}A=C=0, B=D=V_{0} \\
\Delta \rightarrow 0, \widetilde{\Delta} \rightarrow 0, x_{0}=\widetilde{x}_{0}=0 \\
\Lambda=\widetilde{\Lambda}=1, \alpha=\beta\end{array}$ & $V(x)=V_{0}\left[\frac{\theta(-x)}{e^{-\alpha x}}-\frac{\theta(x)}{e^{\alpha x}}\right]$ \\
\hline
\end{tabular}

we need to solve the Schrödinger equation for both regions $x<0$ and $x>0$. Putting (1) in (2) for the left region, we get

$$
\begin{aligned}
& \left\{\frac{d^{2}}{d x^{2}}+2 m\left[E-\frac{A e^{2 \alpha\left(x+x_{0}\right)}}{\left(\Lambda+\Delta e^{\alpha\left(x+x_{0}\right)}\right)^{2}}-\frac{B e^{\alpha\left(x+x_{0}\right)}}{\Lambda+\Delta e^{\alpha\left(x+x_{0}\right)}}\right]\right\} \psi_{L}(x) \\
& =0 .
\end{aligned}
$$

Introducing a new variable $\chi=-(\Delta / \Lambda) e^{\alpha\left(x+x_{0}\right)}$ in (3), one acquires the following equation:

$$
\begin{aligned}
\chi(1-\chi) \frac{d^{2} \psi_{L}(\chi)}{d \chi^{2}}+(1-\chi) \frac{d \psi_{L}(\chi)}{d \chi} & \\
& +\frac{1}{\chi(1-\chi)}\left(a_{1}-a_{2} \chi+a_{3} \chi^{2}\right) \psi_{L}(\chi)=0,
\end{aligned}
$$

where

$$
\begin{gathered}
a_{1}=\frac{2 m E}{\alpha^{2}}, \quad a_{2}=\frac{2 m E}{\alpha^{2}}\left(2 E-\frac{B}{\Delta}\right), \\
a_{3}=\frac{2 m E}{\alpha^{2}}\left(2 E-\frac{A}{\Delta^{2}}-\frac{B}{\Delta}\right),
\end{gathered}
$$

and $\psi_{L}$ is the solution for the left region. In order to get a solution of (4), we take the trial wave function as $\psi_{L}(\chi)=$ $\chi^{\lambda_{1}}(1-\chi)^{\delta_{1}}$ and in that case (4) converts the Gaussian differential equation [48]:

$$
\begin{gathered}
\chi(1-\chi) \frac{d^{2} f(\chi)}{d \chi^{2}}+\left[\left(1+\lambda_{1}\right)-\left(2 \lambda_{1}+2 \delta_{1}+1\right) \chi\right] \frac{d f(\chi)}{d \chi} \\
-\left(\lambda_{1}+\delta_{1}+\gamma_{1}\right)\left(\lambda_{1}+\delta_{1}-\gamma_{1}\right) f(\chi)=0,
\end{gathered}
$$

where

$$
\begin{gathered}
\lambda_{1}=\frac{i k}{\alpha}, \quad k=\sqrt{2 m E} \\
\delta_{1}=\frac{1}{2}\left(1+\sqrt{1+\frac{8 m A}{\alpha^{2} \Delta^{2}}}\right), \quad \gamma_{1}=i \sqrt{\frac{2 m}{\alpha^{2}}\left(E-\frac{A}{\Delta^{2}}-\frac{B}{\Delta}\right)} .
\end{gathered}
$$

The general solution of (6) is given in terms of the Gauss hypergeometric functions as the following form:

$$
\begin{gathered}
f(\chi)=A_{12} F_{1}\left(\lambda_{1}+\delta_{1}-\gamma_{1}, \lambda_{1}+\delta_{1}+\gamma_{1}, 1+2 \lambda_{1} ; \chi\right) \\
+A_{2} \chi_{2}^{-2 \lambda_{1} F_{1}}\left(-\lambda_{1}+\delta_{1}-\gamma_{1},\right. \\
\left.\quad-\lambda_{1}+\delta_{1}+\gamma_{1}, 1-2 \lambda_{1} ; \chi\right) .
\end{gathered}
$$


Therefore, we obtain the complete solution for the left-region $(x<0)$ as

$$
\begin{aligned}
& \psi_{L}(\chi)=A_{1} \chi^{\lambda_{1}}(1-\chi)^{\delta_{1}}{ }_{2} F_{1}( \lambda_{1}+\delta_{1}-\gamma_{1}, \\
&\left.\lambda_{1}+\delta_{1}+\gamma_{1}, 1+2 \lambda_{1} ; \chi\right) \\
&+A_{2} \chi^{-\lambda_{1}}(1-\chi)^{\delta_{1}}{ }_{2} F_{1}\left(-\lambda_{1}+\delta_{1}-\gamma_{1},\right. \\
&\left.-\lambda_{1}+\delta_{1}+\gamma_{1}, 1-2 \lambda_{1} ; \chi\right)
\end{aligned}
$$

Now we search the solution for the right-region $(x>0)$ of the GAMAR type potential. At that rate, (2) turns out to be

$$
\begin{aligned}
& \left\{\frac{d^{2}}{d x^{2}}+2 m\left[E-\frac{C e^{-2 \beta\left(x-\tilde{x}_{0}\right)}}{\left(\widetilde{\Lambda}+\widetilde{\Delta} e^{-\beta\left(x-\widetilde{x}_{0}\right)}\right)^{2}}-\frac{D e^{-\beta\left(x-\tilde{x}_{0}\right)}}{\widetilde{\Lambda}+\widetilde{\Delta} e^{-\beta\left(x-\widetilde{x}_{0}\right)}}\right]\right\} \\
& \quad \times \psi_{R}(x)
\end{aligned}
$$

where $\psi_{R}(x)$ is the right-region solution. If we use a new variable $\eta=-(\widetilde{\Delta} / \widetilde{\Lambda}) e^{-\beta\left(x-\tilde{x}_{0}\right)}$ in (10) we achieve the following equation:

$$
\begin{aligned}
\eta(1-\eta) & \frac{d^{2} \psi_{R}(\eta)}{d \eta^{2}}+(1-\eta) \frac{d \psi_{R}(\eta)}{d \eta} \\
& +\frac{1}{\eta(1-\eta)}\left(b_{1}-b_{2} \eta+b_{3} \eta^{2}\right) \psi_{R}(\eta)=0
\end{aligned}
$$

where

$$
\begin{gathered}
b_{1}=\frac{2 m E}{\beta^{2}}, \quad b_{2}=\frac{2 m E}{\beta^{2}}\left(2 E-\frac{D}{\widetilde{\Delta}}\right), \\
b_{3}=\frac{2 m E}{\beta^{2}}\left(2 E-\frac{C}{\widetilde{\Delta}^{2}}-\frac{D}{\widetilde{\Delta}}\right) .
\end{gathered}
$$

Similarly to the solution that has been suggested for the leftregion $(x<0)$ of the potential, taking the trial wave function $\psi_{R}(\eta)=\eta^{\lambda_{2}}(1-\eta)^{\delta_{2}} h(\eta)$, (11) transforms the Gaussian differential equation. In this way, after a little algebra, the general solution for the right-region is obtained as follows:

$$
\begin{aligned}
& \psi_{R}(\eta)=A_{3} \eta^{\lambda_{2}}(1-\eta)_{2}^{\delta_{2}} F_{1}( \lambda_{2}+\delta_{2}-\gamma_{2} \\
&\left.\lambda_{2}+\delta_{2}+\gamma_{2}, 1+2 \lambda_{2} ; \eta\right) \\
&+A_{4} \eta^{-\lambda_{2}}(1-\eta)^{\delta_{2}}{ }_{2} F_{1}\left(-\lambda_{2}+\delta_{2}-\gamma_{2}\right. \\
&\left.-\lambda_{2}+\delta_{2}+\gamma_{2}, 1-2 \lambda_{2} ; \eta\right)
\end{aligned}
$$

where

$$
\begin{gathered}
\lambda_{2}=\frac{i k}{\beta}, \quad k=\sqrt{2 m E} \\
\delta_{2}=\frac{1}{2}\left(1+\sqrt{1+\frac{8 m C}{\beta^{2} \widetilde{\Delta}^{2}}}\right), \quad \gamma_{2}=i \sqrt{\frac{2 m}{\beta^{2}}\left(E-\frac{A}{\widetilde{\Delta}^{2}}-\frac{B}{\widetilde{\Delta}}\right) .}
\end{gathered}
$$

We have to acquire the asymptotic forms of the solutions taking part in (9) and (13) to calculate the reflection and transmission coefficients. To do it, we need to use the convenient boundary conditions $x \rightarrow-\infty$ and $x \rightarrow+\infty$. For the leftregion $(x<0)$, as $x \rightarrow-\infty, \chi \rightarrow 0$, and $(1-\chi)^{\delta_{1}} \rightarrow 1$, we obtain from (9)

$$
\psi_{L}(x) \sim A_{1}\left(-\frac{\Delta}{\Lambda}\right)^{i(k / \alpha)} e^{i k\left(x+x_{0}\right)}+A_{2}\left(-\frac{\Delta}{\Lambda}\right)^{-i(k / \alpha)} e^{-i k\left(x+x_{0}\right)} .
$$

For the right-region $(x>0)$, as $x \rightarrow+\infty, \eta \rightarrow 0$ and $(1-\eta)^{\delta_{2}} \rightarrow 1$, we get from (13)

$$
\psi_{R}(x) \sim A_{4}\left(-\frac{\tilde{\Delta}}{\widetilde{\Lambda}}\right)^{i(k / \tilde{\beta})} e^{i k\left(x-\tilde{x}_{0}\right)} .
$$

The one-dimensional current density for the Schrödinger equation is defined as follows:

$$
j=\frac{1}{2 m i}\left[\psi(x) \nabla_{x} \psi^{*}(x)-\psi^{*}(x) \nabla_{x} \psi(x)\right] .
$$

Putting the asymptotic behaviors of the obtained solutions for the both regions that are given in (15) and (16) into (17), we achieve the reflection $(R)$ and transmission $(T)$ coefficients, respectively:

$$
\begin{gathered}
R=\frac{j_{\text {ref. }}}{j_{\text {inc. }}}=e^{4 \pi k / \alpha}\left|\frac{A_{2}}{A_{1}}\right|^{2}, \\
T=\frac{j_{\text {trans. }}}{j_{\text {inc. }}}=e^{2 \pi k(1 / \alpha+1 / \beta)}\left|\frac{A_{4}}{A_{1}}\right|^{2},
\end{gathered}
$$

where $j_{\text {ref. }}, j_{\text {inc. }}$, and $j_{\text {trans. }}$ are called reflection, incident, and transmission currents, respectively. To obtain these coefficients clearly, we should use the continuity conditions of the wave function and its derivative given as

$$
\begin{aligned}
& \psi_{L}(x=0)=\psi_{R}(x=0), \\
& \left(\frac{d \psi_{L}}{d x}\right)_{x=0}=\left(\frac{d \psi_{R}}{d x}\right)_{x=0} .
\end{aligned}
$$

By using the above equations, after cumbersome algebra, we come to the following results:

$$
\begin{aligned}
\frac{A_{2}}{A_{1}}=([ & \left.\left(K_{10}+K_{11}\right) H_{3}+K_{12} H_{6}\right] K_{1} H_{1} \\
& \left.-\left[\left(K_{4}+K_{5}\right) H_{1}+K_{6} H_{4}\right] K_{3} H_{3}\right) \\
\times & \left(\left[\left(K_{7}+K_{8}\right) H_{2}+K_{9} H_{8}\right] K_{3} H_{3}\right. \\
& \left.-\left[\left(K_{10}+K_{11}\right) H_{3}+K_{12} H_{6}\right] K_{2} H_{2}\right)^{-1}, \\
\frac{A_{4}}{A_{1}}= & \left(\left[\left(K_{7}+K_{8}\right) H_{2}+K_{9} H_{5}\right] K_{1} H_{1}\right. \\
& \left.-\left[\left(K_{4}+K_{5}\right) H_{1}+K_{6} H_{4}\right] K_{2} H_{2}\right) \\
\times & \left(\left[\left(K_{7}+K_{8}\right) H_{2}+K_{9} H_{8}\right] K_{3} H_{3}\right. \\
& \left.-\left[\left(K_{10}+K_{11}\right) H_{3}+K_{12} H_{6}\right] K_{2} H_{2}\right),
\end{aligned}
$$


TABLE 2: The abbreviations defined in the calculations for the scattering states.

$$
\begin{aligned}
& \sigma=-\frac{\Delta}{\Lambda}, \widetilde{\sigma}=-\frac{\widetilde{\Delta}}{\widetilde{\Lambda}} \\
& K_{1}=\sigma^{\lambda_{1}} e^{\alpha \lambda_{1} x_{0}}\left(1-\sigma e^{\alpha x_{0}}\right)^{\delta_{1}} \\
& K_{2}=\sigma^{-\lambda_{1}} e^{-\alpha \lambda_{1} x_{0}}\left(1-\sigma e^{\alpha x_{0}}\right)^{\delta_{1}} \\
& K_{3}=\tilde{\sigma}^{\lambda_{2}} e^{-\beta \lambda_{2} \tilde{x}_{0}}\left(1-\widetilde{\sigma} e^{\beta \tilde{x}_{0}}\right)^{\delta_{2}} \\
& K_{4}=\sigma^{\lambda_{1}}\left(\alpha \lambda_{1}\right) e^{\alpha \lambda_{1} x_{0}}\left(1-\sigma e^{\alpha x_{0}}\right)^{\delta_{1}} \\
& K_{5}=\sigma^{\lambda_{1}+1}\left(-\alpha \delta_{1}\right) e^{\alpha\left(\lambda_{1}+1\right) x_{0}}\left(1-\sigma e^{\alpha x_{0}}\right)^{\delta_{1}-1} \\
& K_{6}=\sigma^{\lambda_{1}+1}(\alpha) e^{\alpha\left(\lambda_{1}+\right) x_{0}}\left[\frac{\left(\lambda_{1}+\delta_{1}-\gamma_{1}\right)\left(\lambda_{1}+\delta_{1}+\gamma_{1}\right)}{1+2 \lambda_{1}}\right]\left(1-\sigma e^{\alpha x_{0}}\right)^{\delta_{1}} \\
& K_{7}=\sigma^{-\lambda_{1}}\left(-\alpha \lambda_{1}\right) e^{-\alpha \lambda_{1} x_{0}}\left(1-\sigma e^{\alpha x_{0}}\right)^{\delta_{1}} \\
& K_{8}=\sigma^{-\lambda_{1}+1}\left(-\alpha \delta_{1}\right) e^{-\alpha\left(\lambda_{1}+1\right) x_{0}}\left(1-\sigma e^{\alpha x_{0}}\right)^{\delta_{1}-1} \\
& K_{9}=\sigma^{-\lambda_{1}+1}(\alpha) e^{-\alpha\left(\lambda_{1}+1\right) x_{0}}\left[\frac{\left(-\lambda_{1}+\delta_{1}-\gamma_{1}\right)\left(-\lambda_{1}+\delta_{1}+\gamma_{1}\right)}{1-2 \lambda_{1}}\right]\left(1-\sigma e^{\alpha x_{0}}\right)^{\delta_{1}} \\
& K_{10}=\tilde{\sigma}^{\lambda_{2}}\left(\beta \lambda_{2}\right) e^{-\beta \lambda_{2} \tilde{x}_{0}}\left(1-\tilde{\sigma} e^{\beta \tilde{x}_{0}}\right)^{\delta_{2}} \\
& K_{11}=\widetilde{\sigma}^{\lambda_{2}+1}\left(\beta \delta_{2}\right) e^{-\beta\left(\lambda_{2}-1\right) \tilde{x}_{0}}\left(1-\tilde{\sigma} e^{\beta \tilde{x}_{0}}\right)^{\delta_{2}-1} \\
& K_{12}=\tilde{\sigma}^{\lambda_{2}+1}(-\beta) e^{-\beta\left(\lambda_{2}-1\right) \tilde{x}_{0}}\left[\frac{\left(-\lambda_{2}+\delta_{2}-\gamma_{2}\right)\left(-\lambda_{2}+\delta_{2}+\gamma_{2}\right)}{1-2 \lambda_{2}}\right]\left(1-\tilde{\sigma} e^{\beta \tilde{x}_{0}}\right)^{\delta_{2}} \\
& H_{1}={ }_{2} F_{1}\left(\lambda_{1}+\delta_{1}-\gamma_{1}, \lambda_{1}+\delta_{1}+\gamma_{1}, 1+2 \lambda_{1} ; \sigma e^{\alpha x_{0}}\right) \\
& H_{2}={ }_{2} F_{1}\left(-\lambda_{1}+\delta_{1}-\gamma_{1},-\lambda_{1}+\delta_{1}+\gamma_{1}, 1-2 \lambda_{1} ; \sigma e^{\alpha x_{0}}\right) \\
& H_{3}={ }_{2} F_{1}\left(-\lambda_{2}+\delta_{2}-\gamma_{2},-\lambda_{2}+\delta_{2}+\gamma_{2}, 1-2 \lambda_{2} ; \widetilde{\sigma} e^{\alpha \tilde{x}_{0}}\right) \\
& H_{4}={ }_{2} F_{1}\left(\lambda_{1}+\delta_{1}-\gamma_{1}+1, \lambda_{1}+\delta_{1}+\gamma_{1}+1,2+2 \lambda_{1} ; \sigma e^{\alpha x_{0}}\right) \\
& H_{5}={ }_{2} F_{1}\left(-\lambda_{1}+\delta_{1}-\gamma_{1}+1,-\lambda_{1}+\delta_{1}+\gamma_{1}+1,2-2 \lambda_{1} ; \sigma e^{\alpha x_{0}}\right) \\
& H_{6}={ }_{2} F_{1}\left(-\lambda_{2}+\delta_{2}-\gamma_{2}+1,-\lambda_{2}+\delta_{2}+\gamma_{2}+1,2-2 \lambda_{2} ; \widetilde{\sigma} e^{\alpha \tilde{x}_{0}}\right)
\end{aligned}
$$

where the explicit forms of the abbreviations are given in Table 2.

\section{Bound State Solutions and Condition for Energy Eigenvalues}

The aim of this section is to obtain a relation for the energy eigenvalues. If the shape of the potential is a potential well, the bound states occurred. The GAMAR type potential converted the potential well if the parameters are selected such as any of the following options:

(i) $A \rightarrow-A, B \rightarrow-B, C \rightarrow-C$, and $D \rightarrow-D$,

(ii) $A>0,|B| \geq A, B \rightarrow-B$, and $D \rightarrow-D$,

(iii) $B>0,|A| \gg B, A \rightarrow-A$, and $C \rightarrow-C$,

(iv) $B>0, B>A, \Delta \rightarrow-\Delta, \widetilde{\Delta} \rightarrow-\widetilde{\Delta}, \Lambda \rightarrow-\Lambda$, and $\widetilde{\Lambda} \rightarrow-\widetilde{\Lambda}$.

Considering the first option, (2) for $x<0$ yields

$$
\begin{aligned}
& \left\{\frac{d^{2}}{d x^{2}}+2 m\left[E+\frac{A e^{2 \alpha\left(x+x_{0}\right)}}{\left(\Lambda+\Delta e^{\alpha\left(x+x_{0}\right)}\right)^{2}}+\frac{B e^{\alpha\left(x+x_{0}\right)}}{\Lambda+\Delta e^{\alpha\left(x+x_{0}\right)}}\right]\right\} \\
& \times \psi_{L}(x)=0 .
\end{aligned}
$$

Taking the new variable $\chi=-(\Delta / \Lambda) e^{\alpha\left(x+x_{0}\right)}$ and setting the trial wave function $\psi_{L}(\chi)=\chi^{\lambda_{3}}(1-\chi)^{\delta_{3}}$ in $(21)$, it reduces to the Gaussian differential equation. Hence, the general solution for $x<0$ becomes

$$
\begin{aligned}
& \psi_{L}(\chi)= A_{5} \chi^{\lambda_{3}}(1-\chi)^{\delta_{3}}{ }_{2} F_{1}\left(\lambda_{3}+\delta_{3}-\gamma_{3},\right. \\
&\left.\lambda_{3}+\delta_{3}+\gamma_{3}, 1+2 \lambda_{3} ; \chi\right) \\
&+A_{6} \chi^{-\lambda_{3}}(1-\chi)^{\delta_{3}}{ }_{2} F_{1}\left(-\lambda_{3}+\delta_{3}-\gamma_{3},\right. \\
&\left.-\lambda_{3}+\delta_{3}+\gamma_{3}, 1-2 \lambda_{3} ; \chi\right)
\end{aligned}
$$

where

$$
\begin{gathered}
\lambda_{3}=\frac{k}{\alpha}, \quad k=\sqrt{2 m E} \\
\delta_{3}=\frac{1}{2}\left(1+\sqrt{1-\frac{8 m A}{\alpha^{2} \Delta^{2}}}\right), \quad \gamma_{3}=i \sqrt{\frac{2 m}{\alpha^{2}}\left(E+\frac{A}{\Delta^{2}}+\frac{B}{\Delta}\right)} .
\end{gathered}
$$

For $x>0,(2)$ turns into

$$
\begin{aligned}
& \left\{\frac{d^{2}}{d x^{2}}+2 m\left[E+\frac{C e^{-2 \beta\left(x-\tilde{x}_{0}\right)}}{\left(\widetilde{\Lambda}+\widetilde{\Delta} e^{-\beta\left(x-\widetilde{x}_{0}\right)}\right)^{2}}+\frac{D e^{-\beta\left(x-\tilde{x}_{0}\right)}}{\widetilde{\Lambda}+\widetilde{\Delta} e^{-\beta\left(x-\tilde{x}_{0}\right)}}\right]\right\} \\
& \quad \times \psi_{R}(x) .
\end{aligned}
$$


TABLE 3: The abbreviations defined in the calculations for the bound states.

$$
\begin{aligned}
& \sigma=-\frac{\Delta}{\Lambda}, \widetilde{\sigma}=-\frac{\widetilde{\Delta}}{\widetilde{\Lambda}} \\
& K_{13}=\sigma^{\lambda_{3}} e^{\alpha \lambda_{3} x_{0}}\left(1-\sigma e^{\alpha x_{0}}\right)^{\delta_{3}} \\
& K_{14}=\widetilde{\sigma}^{\lambda_{4}} e^{\beta \lambda_{4} \tilde{x}_{0}}\left(1-\widetilde{\sigma} e^{\beta \tilde{x}_{0}}\right)^{\delta_{4}} \\
& K_{15}=\sigma^{\lambda_{3}}\left(\alpha \lambda_{3}\right) e^{\alpha \lambda_{3} x_{0}}\left(1-\sigma e^{\alpha x_{0}}\right)^{\delta_{3}} \\
& K_{16}=\sigma^{\lambda_{3}+1}\left(-\alpha \delta_{3}\right) e^{\alpha\left(\lambda_{3}+1\right) x_{0}}\left(1-\sigma e^{\alpha x_{0}}\right)^{\delta_{3}-1} \\
& K_{17}=\sigma^{\lambda_{3}+1}(\alpha) e^{\alpha\left(\lambda_{3}+1\right) x_{0}}\left(1-\sigma e^{\alpha x_{0}}\right)^{\delta_{3}}\left[\frac{\left(\lambda_{3}+\delta_{3}-\gamma_{3}\right)\left(\lambda_{3}+\delta_{3}+\gamma_{3}\right)}{1+2 \lambda_{3}}\right] \\
& K_{18}=\widetilde{\sigma}^{\lambda_{4}}\left(-\beta \lambda_{4}\right) e^{\beta \lambda_{4} \tilde{x}_{0}}\left(1-\widetilde{\sigma} e^{\beta \tilde{x}_{0}}\right)^{\delta_{4}} \\
& K_{19}=\widetilde{\sigma}^{\lambda_{4}+1}\left(-\beta \delta_{4}\right) e^{\beta\left(\lambda_{4}+1\right) \tilde{x}_{0}}\left(1-\widetilde{\sigma} e^{\beta \tilde{x}_{0}}\right)^{\delta_{4}-1} \\
& K_{20}=\widetilde{\sigma}^{\lambda_{4}+1}(-\beta) e^{\beta\left(\lambda_{4}+1\right) \tilde{x}_{0}}\left(1-\widetilde{\sigma} e^{\beta \tilde{x}_{0}}\right)^{\delta_{4}}\left[\frac{\left(\lambda_{4}+\delta_{4}-\gamma_{4}\right)\left(\lambda_{4}+\delta_{4}+\gamma_{4}\right)}{1+2 \lambda_{4}}\right] \\
& H_{7}={ }_{2} F_{1}\left(\lambda_{3}+\delta_{3}-\gamma_{3}, \lambda_{3}+\delta_{3}+\gamma_{3}, 1+2 \lambda_{3} ; \sigma e^{\alpha x_{0}}\right) \\
& H_{8}={ }_{2} F_{1}\left(\lambda_{4}+\delta_{4}-\gamma_{4}, \lambda_{4}+\delta_{4}+\gamma_{4}, 1+2 \lambda_{4} ; \widetilde{\sigma} e^{\alpha \tilde{x}_{0}}\right) \\
& H_{9}={ }_{2} F_{1}\left(\lambda_{3}+\delta_{3}-\gamma_{3}+1, \lambda_{3}+\delta_{3}+\gamma_{3}+1,2+2 \lambda_{3} ; \sigma e^{\alpha x_{0}}\right) \\
& H_{10}={ }_{2} F_{1}\left(\lambda_{4}+\delta_{4}-\gamma_{4}+1, \lambda_{4}+\delta_{4}+\gamma_{4}+1,2+2 \lambda_{4} ; \widetilde{\sigma} e^{\alpha \tilde{x}_{0}}\right)
\end{aligned}
$$

By using the transform $\eta=-(\widetilde{\Delta} / \widetilde{\Lambda}) e^{-\beta\left(x-\widetilde{x}_{0}\right)}$ and organizing the trial wave function $\psi_{R}(\eta)=\eta^{\lambda_{4}}(1-\eta)^{\delta_{4}} h(\eta)$ in (24), the general solution for the right-region of the potential $(x>0)$ is obtained in the following form:

$$
\begin{aligned}
& \psi_{R}(\eta)=A_{7} \eta^{\lambda_{4}}(1-\eta)^{\delta_{4}}{ }_{2} F_{1}( \lambda_{4}+\delta_{4}-\gamma_{4}, \\
&\left.\lambda_{4}+\delta_{4}+\gamma_{4}, 1+2 \lambda_{4} ; \eta\right) \\
&+A_{8} \eta^{-\lambda_{4}}(1-\eta)^{\delta_{4}}{ }_{2} F_{1}\left(-\lambda_{4}+\delta_{4}-\gamma_{4},\right. \\
&\left.-\lambda_{4}+\delta_{4}+\gamma_{4}, 1-2 \lambda_{4} ; \eta\right),
\end{aligned}
$$

where

$$
\begin{gathered}
\lambda_{4}=\frac{k}{\beta}, \quad k=\sqrt{2 m E} \\
\delta_{4}=\frac{1}{2}\left(1+\sqrt{1-\frac{8 m C}{\beta^{2} \widetilde{\Delta}^{2}}}\right), \quad \gamma_{4}=i \sqrt{\frac{2 m}{\beta^{2}}\left(E+\frac{A}{\widetilde{\Delta}^{2}}+\frac{B}{\widetilde{\Delta}}\right)} .
\end{gathered}
$$

In order to obtain a regular wave function from (22) and (25), we should set $A_{6}=A_{8}=0$ and we obtain

$$
\begin{gathered}
\psi_{L}(\chi)=A_{5} \chi^{\lambda_{3}}(1-\chi)_{2}^{\delta_{3}} F_{1}\left(\lambda_{3}+\delta_{3}-\gamma_{3},\right. \\
\left.\lambda_{3}+\delta_{3}+\gamma_{3}, 1+2 \lambda_{3} ; \chi\right), \\
\psi_{R}(\eta)=A_{7} \eta^{\lambda_{4}}(1-\eta)^{\delta_{4}}{ }_{2} F_{1}\left(\lambda_{4}+\delta_{4}-\gamma_{4},\right. \\
\left.\lambda_{4}+\delta_{4}+\gamma_{4}, 1+2 \lambda_{4} ; \eta\right) .
\end{gathered}
$$

Then, using boundary conditions which are given in (19), we get the energy eigenvalues equation for the bound states as follows:

$$
\begin{aligned}
& \left\{\left[\left(K_{18}+K_{19}\right) K_{13}-\left(K_{15}+K_{16}\right) K_{14}\right] H_{8}+K_{13} K_{20} H_{10}\right\} H_{7} \\
& -K_{14} K_{17} H_{8} H_{9}=0,
\end{aligned}
$$

where the abbreviations are given in Table 3.

\section{Discussion and Conclusion}

In this study, we solve the one-dimensional Schrödinger equation, which is the fundamental equation of the quantum mechanic, with the GAMAR type potential and we achieve the wave functions describing the bound and scattering states in terms of hypergeometric functions within the framework of the change of variable method. The reflection and transmission coefficients are obtained by using the asymptotic behavior of the scattering states wave functions as well as to continuity conditions. We also derive a relation for the bound state which is described by the energy eigenvalues and defined as the energy eigenvalue condition. Further, we show how the transmission and reflection coefficients depend on the GAMAR type potential by using Mathematica Software package.

As shown in Figure 2, the unitary condition $(T+R=1)$ is satisfied certainly for the different values of the potential parameters: the transmission coefficient approaches unity asymptotically for increasing the energy value and the reflection coefficient goes to zero asymptotically for decreasing values of the energy. The left and right illustrations of Figure 3 show effect of parameters $\Delta, \widetilde{\Delta}$ on the transmission coefficient according to the energy and also potential strength, respectively. According to left illustration, the transmission 


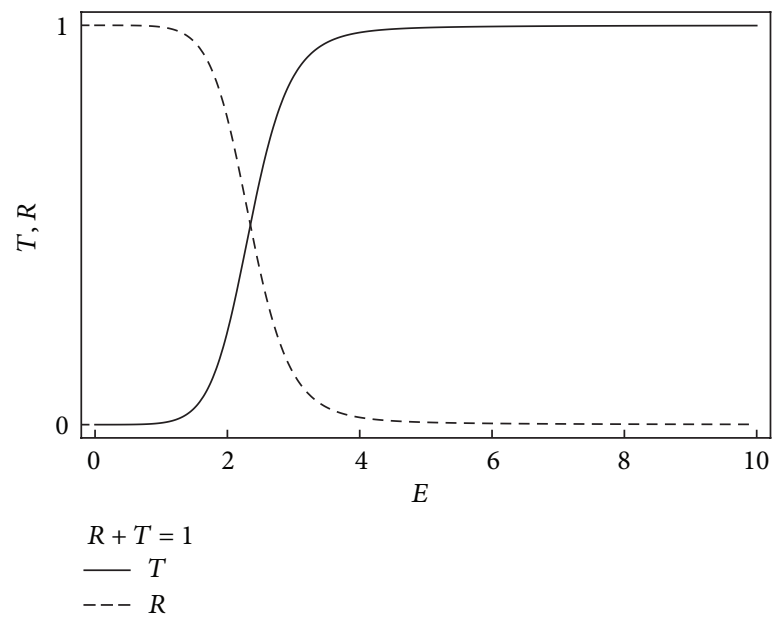

FIgURE 2: The unitarity condition. The transmission $T$ and reflection $R$ coefficient for the GAMAR type potential barrier varying with $E$ for $\alpha=\beta=2, x_{0}=\widetilde{x}_{0}=1, \Lambda=\widetilde{\Lambda}=1, \Delta=\widetilde{\Delta}=2, A=4, C=5, B=D=3$, and $m=1$.
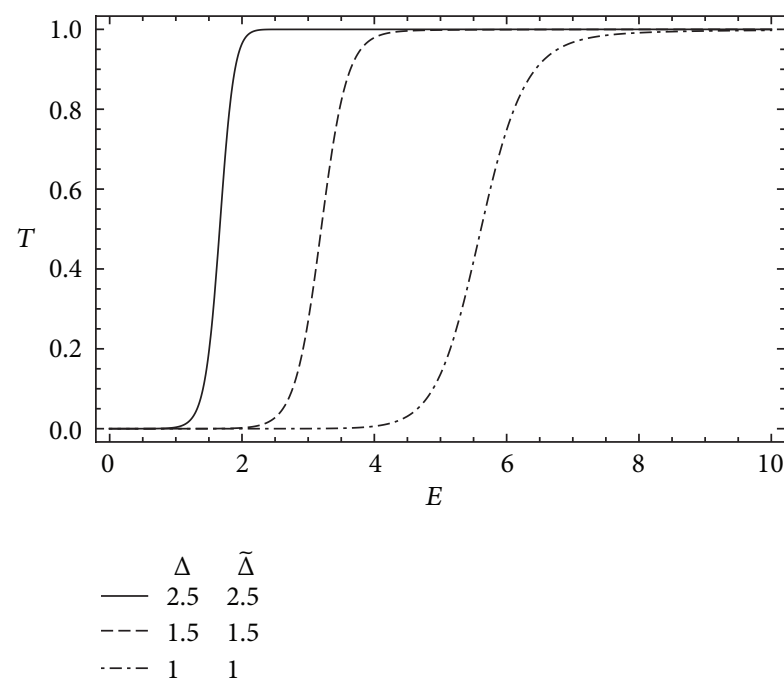
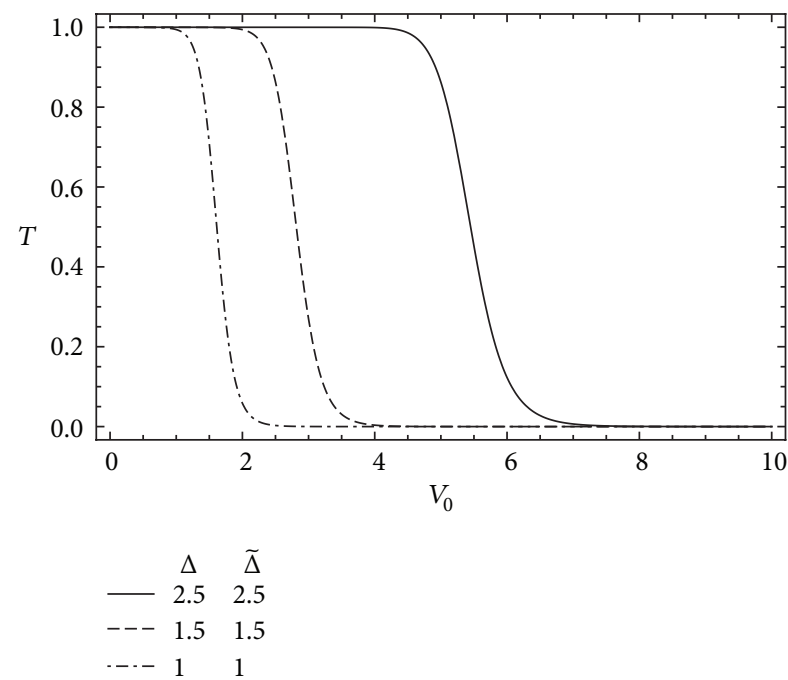

(b)

FIgURE 3: The effect of the GAMAR type potential parameters $(\Delta, \widetilde{\Delta})$ on the transmission coefficient for $\alpha x_{0}=2, \beta \widetilde{x}_{0}=4$ (lower values of the $\alpha x_{0}, \beta \tilde{x}_{0}$ ). (a) shows $T$ for varying $E$ with $\alpha=\beta=2, x_{0}=1, \widetilde{x}_{0}=2, \Lambda=\widetilde{\Lambda}=1, A=B=C=D=3$, and $m=1$. (b) displays $T$ for varying $V_{0}$ with $\alpha=\beta=2, x_{0}=1, \tilde{x}_{0}=2, \Lambda=\widetilde{\Lambda}=1, A=B=C=D=V_{0}, E=3$, and $m=1$.

probability of the Schrödinger particle from the GAMAR type potential barrier takes place at a lower energy value for increasing values of the parameters $\Delta, \widetilde{\Delta}$. In the right illustration, the transmission probability of the particle exactly gets the value of one if the potential strength $\left(V_{0}\right)$ is zero and it gets the value of zero at the higher values of the potential strength for decreasing values of the parameters $\Delta, \widetilde{\Delta}$. It is seen in Figure 4, if we increase the numerical values of the $\alpha x_{0}$ and $\beta \tilde{x}_{0}$ which are stayed as a multiplier in the denominator of the potential function, the transmission resonances occur. One can clearly see from left illustration of Figure 4 that the transmission coefficient goes to one; the condition provides $E \sim\left[(A+B \Delta) / \Delta^{2}\right]$, where $\Delta=\widetilde{\Delta}$.
In the right illustration, transmission coefficient approaches zero; the condition must be $V_{0} \sim\left[\Delta^{2} /(\Delta+1)\right]$, where $A=$ $B=C=D=V_{0}$. Figures 5 and 6 display the transmission probability of Schrödinger particles from the potentials which can be derived from the GAMAR type potential. As shown in Figure 5, the transmission coefficient of Schrödinger particles goes to unity for the GAWS potential at lower values of energy, while it goes to unity for the ASH potential at higher values of energy. Similarly, in Figure 6, the transmission coefficient goes to unity for the Woods-Saxon potential at lower values of energy, while it goes to unity for the Hulthen potential at higher values of energy. From Figure 5, we see that our results are consistent with the results 


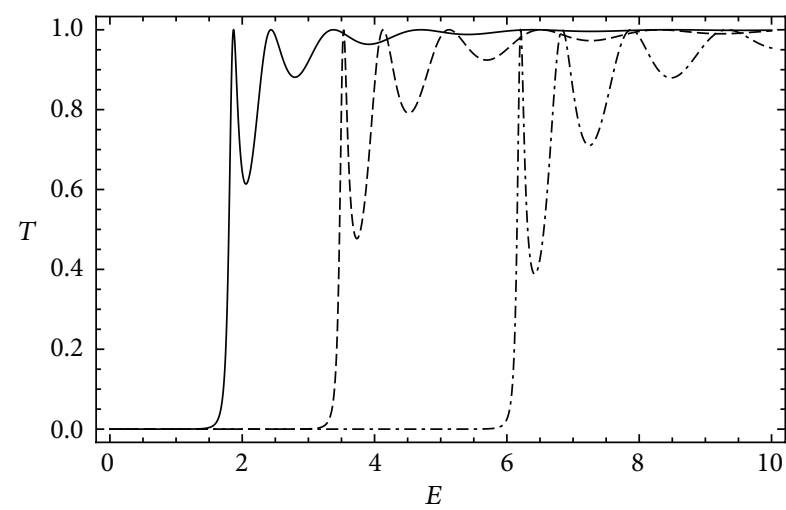

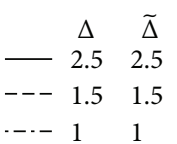
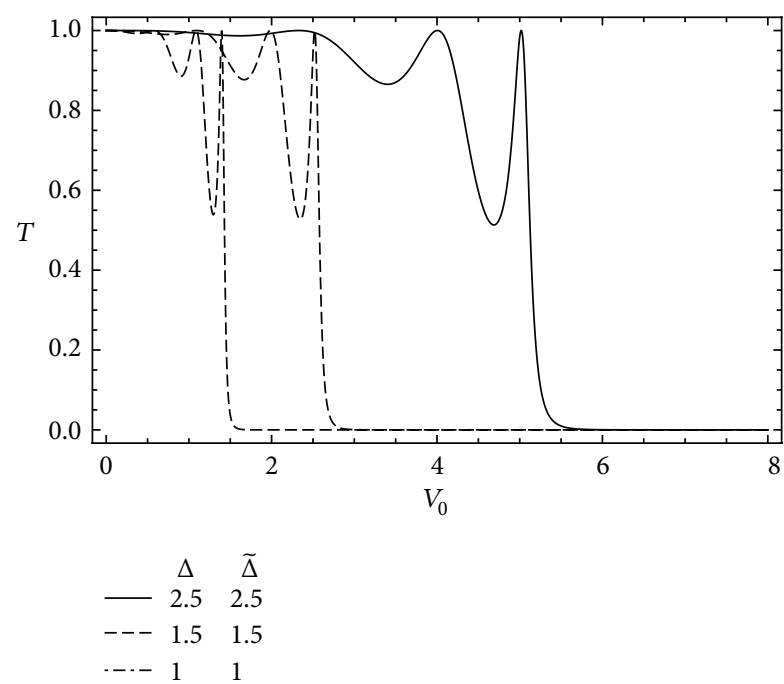

(b)

FiguRE 4: The effect of the GAMAR type potential parameters $(\Delta, \widetilde{\Delta})$ on the transmission coefficient for $\alpha x_{0}=20, \beta \tilde{x}_{0}=30$ (large values of the $\alpha x_{0}, \beta \widetilde{x}_{0}$ ). (a) shows $T$ for varying $E$ with $\alpha=\beta=10, x_{0}=2, \widetilde{x}_{0}=3, \Lambda=\widetilde{\Lambda}=1, A=B=C=D=3$, and $m=1$. (b) shows $T$ for varying $V_{0}$ with $\alpha=\beta=10, x_{0}=2, \widetilde{x}_{0}=3, \Lambda=\widetilde{\Lambda}=1, A=B=C=D=V_{0}, E=3$, and $m=1$.

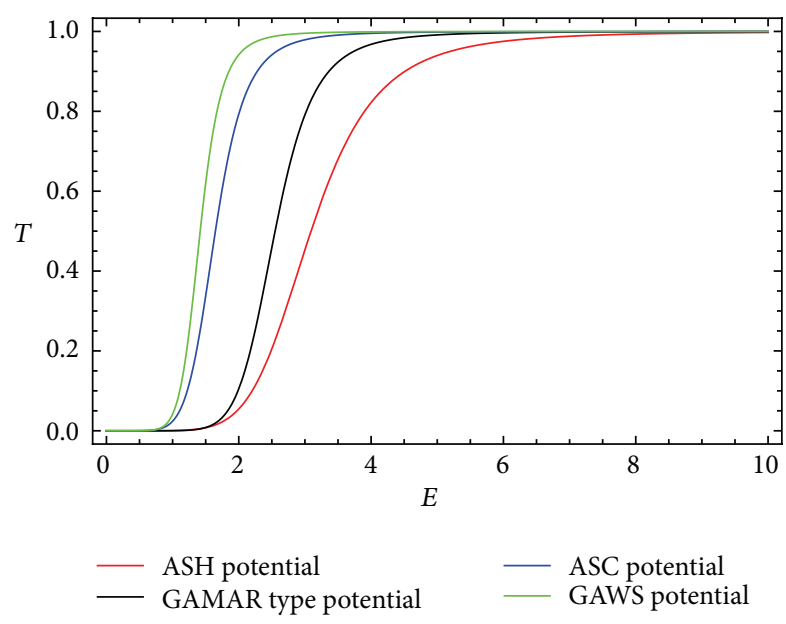

FIGURE 5: The effect of the asymmetric potentials on the transmission coefficient: the ASH potential for $\alpha=0.5, \beta=0.8, x_{0}=\tilde{x}_{0}=0$, $\Lambda=\widetilde{\Lambda}=1, \Delta=\widetilde{\Delta}=-0.5, A=C=0, B=D=2$, and $m=1$, the GAMAR potential for $\alpha=0.5, \beta=0.8, x_{0}=\widetilde{x}_{0}=0.5, \Lambda=\widetilde{\Lambda}=1$, $\Delta=\widetilde{\Delta}=0.5, A=B=C=D=2$, and $m=1$, the ASC potential for $\alpha=0.5, \beta=0.8, x_{0}=\widetilde{x}_{0}=0, \Lambda=\widetilde{\Lambda}=1, \Delta \rightarrow 0, \widetilde{\Delta} \rightarrow 0$, $A=C=0, B=D=2$, and $m=1$, and the GAWS potential for $\alpha=0.5, \beta=0.8, x_{0}=\widetilde{x}_{0}=0.5, \Lambda=\widetilde{\Lambda}=1, \Delta=\widetilde{\Delta}=0.5, A=C=0$, $B=D=2$, and $m=1$.

of the work of Arda et al. [28] for the ASH potential and it is also compatible with [49] for the GAWS potential.

\section{Conflict of Interests}

The authors declare that there is no conflict of interests regarding the publication of this paper.

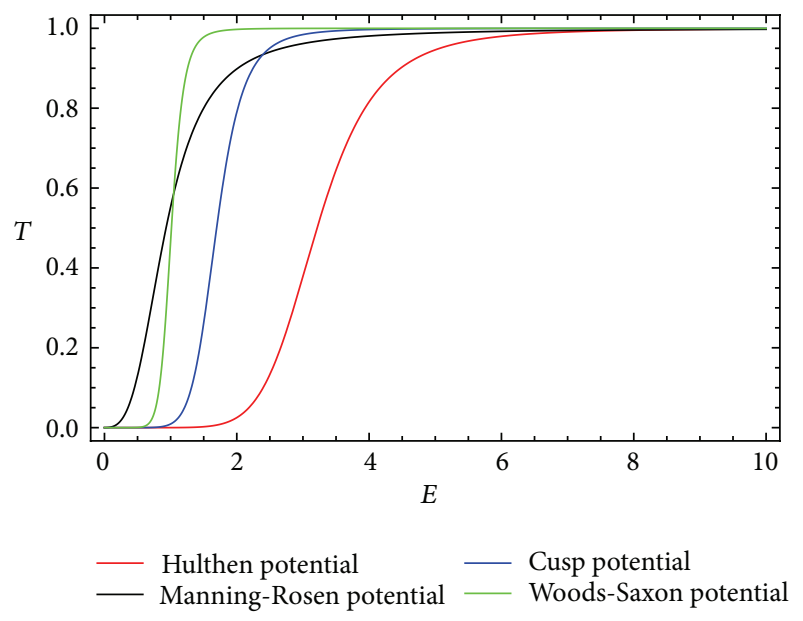

FIGURE 6: The effect of the potentials on the transmission coefficient: the Hulthen potential for $\alpha=\beta=0.5, x_{0}=\widetilde{x}_{0}=0, \Lambda=\widetilde{\Lambda}=1$, $\Delta=\widetilde{\Delta}=-0.5, A=C=0, B=D=2$, and $m=1$, the ManningRosen potential for $\alpha=\beta=0.5, x_{0}=\widetilde{x}_{0}=0, \Lambda=\widetilde{\Lambda}=1, \Delta \rightarrow-1$, $\widetilde{\Delta} \rightarrow-1, A=B=0, C=D=2$, and $m=1$, the Cusp potential for $\alpha=\beta=0.5, x_{0}=\tilde{x}_{0}=0, \Lambda=\widetilde{\Lambda}=1, \Delta \rightarrow 0, \widetilde{\Delta} \rightarrow 0$, $A=C=0, B=D=2$, and $m=1$, and the Woods-Saxon potential for $\alpha=\beta=0.5, x_{0}=\widetilde{x}_{0}=0.5, \Lambda=\widetilde{\Lambda}=1, \Delta=\widetilde{\Delta}=0.5, A=C=0$, $B=D=2$, and $m=1$.

\section{Acknowledgments}

This work was supported by Mersin University Scientific Research Unit (BAP-FBE F (AT) 2012-2 YL). The author (Ahmet Taş) wishes to thank for its support. 


\section{References}

[1] A. Kratzer, "Die ultraroten rotationsspektren der halogenwasserstoffe," Zeitschrift für Physik A Hadrons and Nuclei, vol. 3, no. 5, pp. 289-307, 1920.

[2] P. M. Morse, "Diatomic molecules according to the wave mechanics. II. Vibrational levels," Physical Review, vol. 34, p. 57, 1929.

[3] C. Eckart, "The penetration of a potential barrier by electrons," Physical Review, vol. 35, no. 11, pp. 1303-1309, 1930.

[4] N. Rosen and P. M. Morse, "On the vibrations of polyatomic molecules," Physical Review, vol. 42, no. 2, pp. 210-217, 1932.

[5] M. F. Manning and N. Rosen, "Minutes of the Middletown meeting, October 14, 1933," Physical Review, vol. 44, no. 11, pp. 951-954, 1933.

[6] G. Pöschl and E. Teller, "Bemerkungen zur Quantenmechanik des anharmonischen Oszillators," Zeitschrift für Physik, vol. 83, no. 3-4, pp. 143-151, 1933.

[7] L. Hulthen, "Uber die Eigenlösungen der Schrödinger chung des Deutrons," Arkiv för Matematik, Astronomi och Fysik A, vol. 28 , no. $5,1942$.

[8] R. Woods D and D. S. Saxon, "Diffuse surface optical model for nucleon-nuclei scattering," Physical Review, vol. 95, no. 2, pp. 577-578, 1954.

[9] F. L. Scarf, "New soluble energy band problem," Physical Review, vol. 112, pp. 1137-1140, 1958.

[10] P.-Q. Wang, L.-H. Zhang, C.-S. Jia, and J.-Y. Liu, "Equivalence of the three empirical potential energy models for diatomic molecules," Journal of Molecular Spectroscopy, vol. 274, pp. 5-8, 2012.

[11] T. Chen, S.-R. Lin, and C.-S. Jia, "Solutions of the Klein-Gordon equation with the improved Rosen-Morse potential energy model," The European Physical Journal Plus, vol. 128, p. 69, 2013.

[12] C. Jia, T. Chen, and S. He, "Bound state solutions of the Klein-Gordon equation with the improved expression of the Manning-Rosen potential energy model," Physics Letters A, vol. 377, no. 9, pp. 682-686, 2013.

[13] V. M. Villalba and W. Greiner, "Transmission resonances and supercritical states in a one-dimensional cusp potential," Physical Review A, vol. 67, Article ID 052707, 2003.

[14] S. Flugge, Practical Quantum Mechanics, Springer, New York, NY, USA, 2nd edition, 1994.

[15] R. G. Newton, Scattering Theory of Waves and Particles, Springer, New York, NY, USA, 2nd edition, 1982.

[16] L. D. Landau and E. M. Lifshitz, Quantum Mechanics NonRelativistic Theory, Pergamon Press, New York, NY, USA, 3rd edition, 1977.

[17] C. Y. Chen, F. L. Lu, and D. S. Sun, "Exact solutions of scattering states for the S-wave Schrödinger equation with the ManningRosen potential," Physica Script, vol. 76, no. 5, pp. 428-430, 2007.

[18] X.-T. Hu, L-H. Zhang, and C.-S. Jia, "D-dimensional energies for lithium dimer," Journal of Molecular Spectroscopy, vol. 297, pp. 21-24, 2014.

[19] J.-Y. Liu, G.-D. Zhang, and C.-S. Jia, "Calculation of the interaction potential energy curve and vibrational levels for the $\sum_{2}^{+}$source state of ${ }^{7} \mathrm{Li}_{2}$ source molecule," Physics Letters A, vol. 377, no. 21-22, pp. 1444-1447, 2013.

[20] G. Wei, C. Long, and S. Dong, "The scattering of the ManningRosen potential with centrifugal term," Physics Letters. A, vol. 372, no. 15, pp. 2592-2596, 2008.

[21] S. Meyur and S. Debnath, "Solution of the Schrödinger equation with Hulthén plus Manning-Rosen potential," Latin-American Journal of Physics Education, vol. 3, no. 2, 2009.
[22] W. Qiang and S. H. Dong, "The Manning-Rosen potential studied by a new approximate scheme to the centrifugal term," Physica Scripta, vol. 79, no. 4, Article ID 045004, 5 pages, 2009.

[23] S. A. S. Ahmed and L. Buragohain, "Exactly solved potentials generated from the Manning-Rosen potential using extended transformation method," Electronic Journal of Theoretical Physics, vol. 23, no. 3, pp. 145-154, 2010.

[24] A. D. Antia, A. N. Ikot, and L. E. Akpabio, "Exact solutions of the Schrodinger equation with Manning-Rosen potential plus a ring-shaped like potential by Nikiforov-Uvarov method," European Journal of Scientific Research, vol. 46, no. 1, pp. 107-118, 2010.

[25] A. N. Ikot, L. E. Akpabio, and E. B. Umoren, "Exact solution of Schrödinger equation with inverted woods-saxon and Manning-Rosen potentials," Journal of Scientific Research, vol. 3, no. 1, pp. 25-33, 2011.

[26] S. M. Ikhdair and R. Sever, "Approximate $I$-state solutions of the $D$-dimensional Schrödinger equation for Manning-Rosen potential," Annalen der Physik, vol. 17, no. 11, pp. 897-910, 2008.

[27] W. C. Qiang, Y. Gao, and R. S. Zhou, "Arbitrary/-state approximate solutions of the Hulthén potential through the exact quantization rule," Central European Journal of Physics, vol. 6, no. 2, pp. 356-362, 2008.

[28] A. Arda, O. Aydogdu, and R. Sever, "Scattering and bound state solutions of the asymmetric Hulthén potential," Physica Scripta, vol. 84, no. 2, Article ID 025004, 2011.

[29] O. Bayrak and I. Boztosun, "Bound state solutions of the Hulthén potential by using the asymptotic iteration method," Physica Scripta, vol. 76, no. 1, pp. 92-96, 2007.

[30] C. Berkdemir, A. Berkdemir, and R. Sever, "Polynomial solutions of the Schrödinger equation for the generalized WoodsSaxon potential," Physical Review C, vol. 72, article 027001, 2005.

[31] J. Sadeghi, "Factorization method and solution of the noncentral modified kratzer potential," Acta Physica Polonica A, vol. 112, no. 1, pp. 23-28, 2007.

[32] A. Arda, O. Aydoğdu, and R. Sever, "Scattering of the WoodsSaxon potential in the SCHrödinger equation," Journal of Physics A: Mathematical and Theoretical, vol. 43, no. 42, Article ID 425204, 2010.

[33] D. Agboola, "Solutions to the modified Pöschl-Teller potential in D-dimensions," Chinese Physics Letters, vol. 27, no. 4, Article ID 040301, 2010.

[34] W. C. Qiang, W. L. Chen, and G. F. Wei, “The scattering states of the $l$-wave Schrödinger equation with the second Pöschl-Tellerlike potential," Physica Scripta, vol. 79, no. 2, Article ID 025005, 6 pages, 2009.

[35] M. Hamzavi and S. M. Ikhdair, "Approximate $l$-state solution of the trigonometric Pöschl-Teller potential," Molecular Physics, vol. 110, no. 24, pp. 3031-3039, 2012.

[36] C. Tezcan and R. Sever, "Exact solutions of the Schrödinger equation with position-dependent effective mass via general point canonical transformation," Journal of Mathematical Chemistry, vol. 42, no. 3, pp. 387-395, 2007.

[37] W. Qiang and S. Dong, "Arbitrary $l$-state solutions of the rotating Morse potential through the exact quantization rule method," Physics Letters. A, vol. 363, no. 3, pp. 169-176, 2007.

[38] C. Berkdemir and J. Han, "Any l-state solutions of the Morse potential through the Pekeris approximation and NikiforovUvarov method," Chemical Physics Letters, vol. 409, no. 4-6, pp. 203-207, 2005. 
[39] M. Znojil, "A generalized Morse asymmetric potential and multiplets of its non-numerical exact bound states," Journal of Physics A, vol. 27, no. 22, pp. 7491-7501, 1994.

[40] O. Bayrak, M. Karakoc, I. Boztosun, and R. Sever, "Analytical solution of the Schrödinger equation for Makarov potential with any $l$ angular momentum," International Journal of Theoretical Physics, vol. 47, no. 11, pp. 3005-3014, 2008.

[41] S. Ikhdair, " On the bound-state solutions of the ManningRosen potential including an improved approximation to the orbital centrifugal term," Physica Scripta, vol. 83, Article ID 015010, 2011.

[42] R. J. le Roy and R. B. Bernstein, "Dissociation energy and longrange potential of diatomic molecules from vibrational spacings of hgher levels," Journal of Chemical Physics, vol. 52, p. 3869, 1970.

[43] J. M. Cai, P. Y. Cai, and A. Inomata, "Path-integral treatment of the Hulthén potential," Physical Review A, vol. 34, no. 6, pp. 4621-4628, 1986.

[44] A. Tas, The scattering and bound states of the Schrodinger particle in GAMAR type potential [M.S. thesis], University of Mersin, 2012.

[45] S. Alpdoğan, O. Aydoğdu, and A. Havare, "Relativistic spinless particles in the generalized asymmetric Woods-Saxon potential," Journal of Physics A: Mathematical and Theoretical, vol. 46, no. 1, Article ID 015301, 13 pages, 2013.

[46] K. Sogut and A. Havare, "Scattering of vector bosons by an asymmetric Hulthen potential," Journal of Physics A: Mathematical and Theoretical, vol. 43, no. 22, Article ID 225204, 2010.

[47] K. Sogut and A. Havare, "Transmission resonances in the Duffin-Kemmer-Petiau equation in $(1+1)$ dimensions for an asymmetric cusp potential," Physica Scripta, vol. 82, Article ID 045013, 2010.

[48] M. Abramowitz and A. Stegun, Handbook of Mathematical Functions, Dover, New York, NY, USA, 1965.

[49] S. Alpdogan, Scattering of vector bosons by generalized WoodsSaxon potential [M.S. thesis], University of Mersin, 2012. 

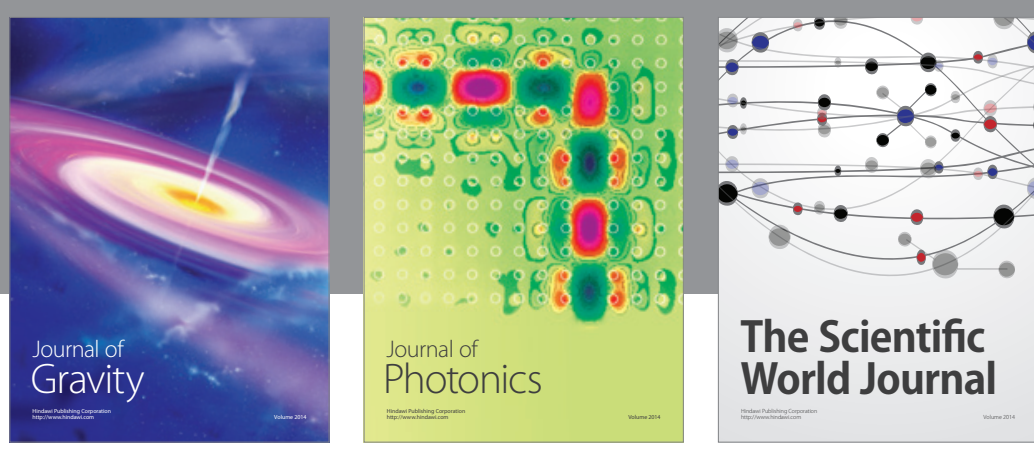

The Scientific World Journal
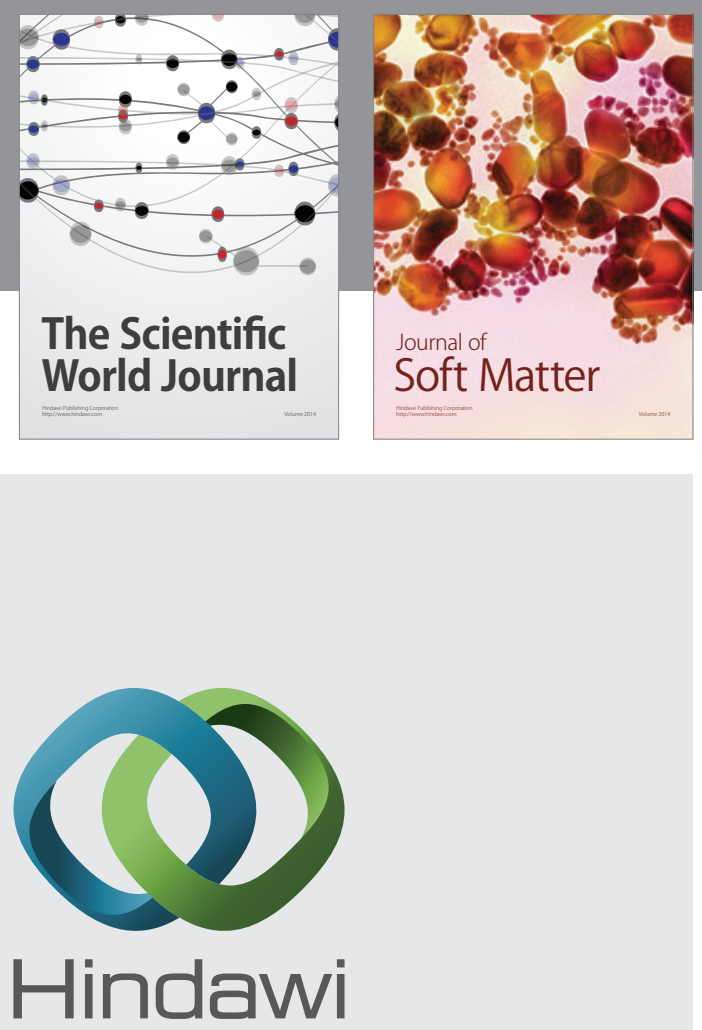

Submit your manuscripts at

http://www.hindawi.com

nternational Journal of

Statistical Mechanics
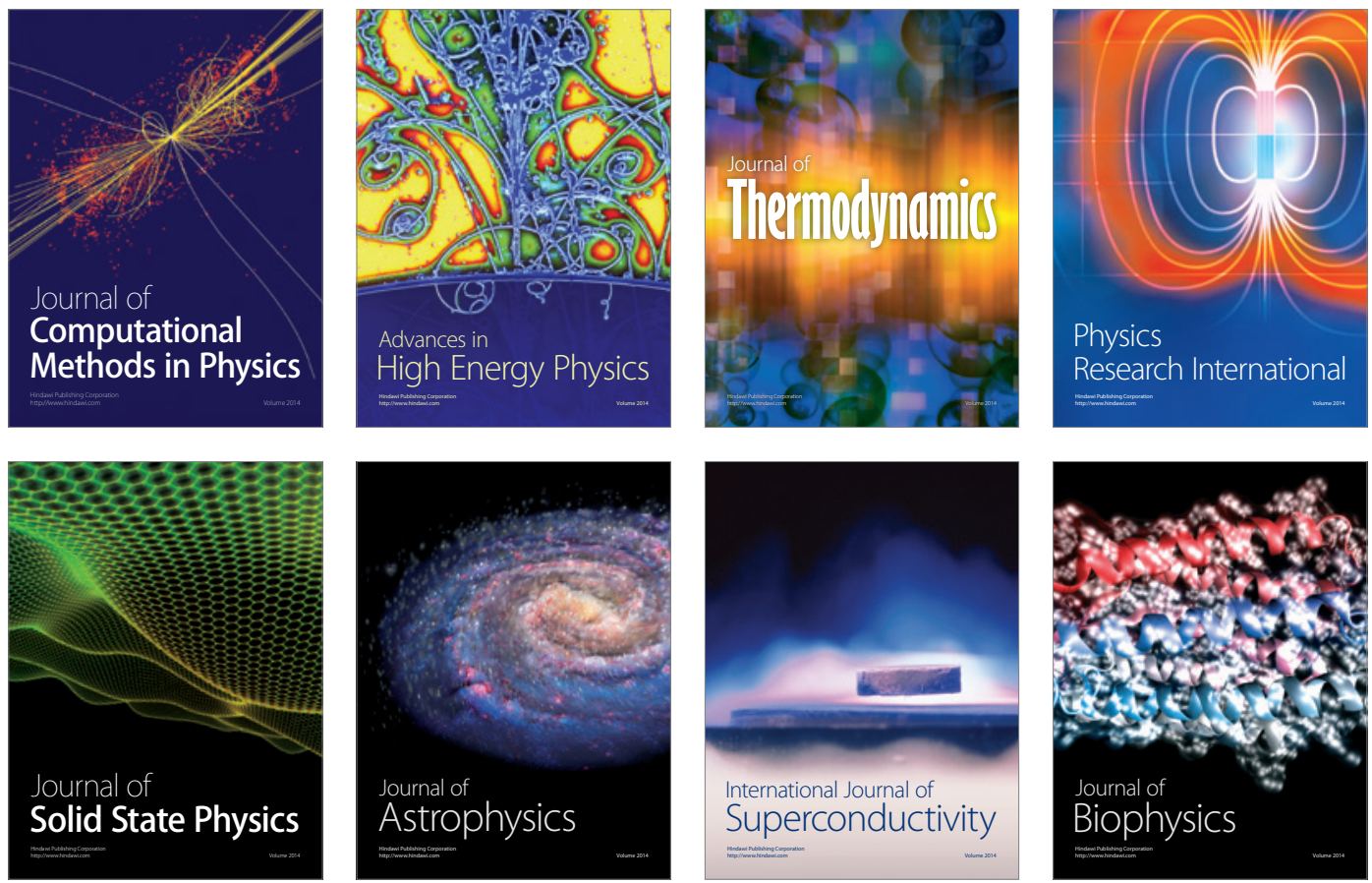
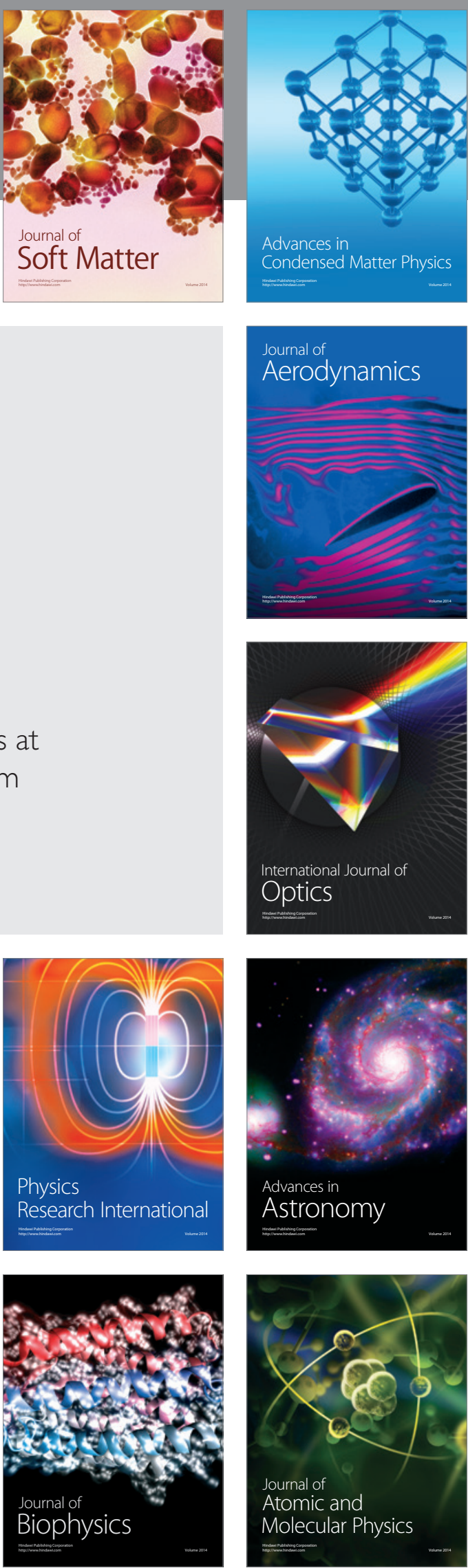